\title{
Microscopic derivation of the hydrodynamic equations for the superfluid fermi-systems
}

\author{
P.Shygorin, A.Svidzynsky \\ Lesya Ukrainka's Volyn State University
}

Received August 3, 2005

\begin{abstract}
Starting from the first principles of statistical mechanics, the two-fluid hydrodynamics of superconductor in ideal approximation is constructed. The system of equations of motion for normal and anomalous correlation functions is used for hydrodynamic description. Transition to equations of hydrodynamics is performed using an expansion of equations of motion for correlation functions in terms of a small parameter.
\end{abstract}

Key words: two-fluid hydrodynamics, correlation function, superfluid Fermi-systems

PACS: 05.30.Fk, 47.37.+q

\section{Introduction}

Due to specific properties the quantum liquids turn out to be a very interesting object in physical studies. They include superfluids He-3 and He-4, a superconducting Fermi-systems, a trapped Bose gas, a core of neutron stars, etc $[1,2]$. In the weakly-nonequilibrium states the equations of hydrodynamics are very important tool of describing the afore-mentioned systems. The phenomena of superconductivity and superfluidity are known to be deeply related. The property of the nondissipative current states, that is caused by the phase transition to a more-organized state, unites them [3]. It is not surprising that the equations of hydrodynamics of these systems have a similar structure (the so-called two-fluid equations). The phenomenological hydrodynamics of superfluid He-4 was constructed by Landau in 1941 [4]. At the microscopic level these equations were derived by Bogoliubov in 1963 [5]. The two-fluid model of superconductor was derived in 1965 by Svidzynsky and Slusarev [6], and independently by Stephen [7]. The system of equations of motion for the normal and the anomalous correlation functions is the starting point for constructing the hydrodynamic equations in $[5,6]$. The transition to equations of hydrodynamics is performed using an expansion of equations of motion for correlation functions in terms of a small parameter. The small parameter is introduced formally. 
This is a noticeable drawback of these studies. In the [5] the so-called "parameter of homogeneity" is chosen as small parameter, while in [6] it is the Plank constant, which obviously was possible to put equal to unity.

In the present paper, where following the chart of work [6], starting from the first principles of statistical mechanics, we derived the equations of two-fluid hydrodynamics of superconductor in an ideal approximation. An electronic liquid in the superconductor is described by the four-fermion Hamiltonian, that reflects a direct interaction between electrons. In terms of the Heisenberg equations we constructed the equations of motion for correlation functions. The mean-field approach is used for disclosure of higher correlation functions. By writing down equations of motion in a dimensionless form it is possible to select a small parameter, which is equal to the ratio of the length of coherence to a characteristic length of changes in macroscopic quantities (such as the mean number of particles, momentum, energy). The expansion in terms of this small parameter coincides with the expansion in terms of gradients of the macroscopic quantities.

\section{Equations of motion for correlation functions}

Let us consider a superconductor in the BCS model. In this model the Hamiltonian of the system of electrons in the presence of an external electromagnetic field in the second quantization representation is as follows (we set $\hbar=c=1$ throughout this paper)

$$
\begin{aligned}
\hat{H}= & \sum_{\sigma} \int \mathrm{d} \vec{r} \Psi_{\sigma}^{+}(\vec{r})\left\{\frac{1}{2 m}(\hat{\vec{p}}-\mathrm{e} \vec{A}(\vec{r}, t))^{2}+\mathrm{e} A_{0}(\vec{r}, t)\right\} \Psi_{\sigma}(\vec{r}) \\
& +g \int \mathrm{d} \vec{r} \Psi_{\uparrow}^{+}(\vec{r}) \Psi_{\downarrow}^{+}(\vec{r}) \Psi_{\downarrow}(\vec{r}) \Psi_{\uparrow}(\vec{r}) .
\end{aligned}
$$

To construct the hydrodynamics of a system with spontaneous broken symmetry we should proceed from the extended system of correlation functions [5], which is formed both by a normal and by an anomalous correlation function. Therefore we will start with a system of correlation functions in the next form

$$
\left\langle\Psi_{\uparrow}^{+}\left(x_{1}\right) \Psi_{\uparrow}\left(x_{2}\right)\right\rangle, \quad\left\langle\Psi_{\downarrow}\left(x_{1}\right) \Psi_{\uparrow}\left(x_{2}\right)\right\rangle .
$$

Here $x_{i} \equiv\left(\vec{r}_{i}, t\right)$, and the dependence of the creation and annihilation operators on time is given through a Heisenberg representation, for instance

$$
\Psi_{\uparrow}^{+}\left(x_{1}\right)=\mathrm{e}^{\mathrm{i} \hat{H} t} \Psi_{\uparrow}^{+}\left(\vec{r}_{1}\right) \mathrm{e}^{-\mathrm{i} \hat{H} t}
$$

the angular brackets indicate an average at the local-equilibrium ensemble.

Using the Heisenberg's equation of motion

$$
\mathrm{i} \frac{\partial \Psi_{\sigma}(x)}{\partial t}=\left[\Psi_{\sigma}(x), \hat{H}\right]_{-}
$$


we obtain the equations of motion for correlation functions (2). These equations are as follows:

$$
\begin{aligned}
& \left\{\mathrm{i} \frac{\partial}{\partial t}+\mathrm{e} A_{0}\left(x_{1}\right)-\mathrm{e} A_{0}\left(x_{2}\right)\right\}\left\langle\Psi_{\uparrow}^{+}\left(x_{1}\right) \Psi_{\uparrow}\left(x_{2}\right)\right\rangle \\
& \quad+\frac{1}{2 m}\left[\left(\hat{\vec{p}}_{1}+\mathrm{e} \vec{A}\left(x_{1}\right)\right)^{2}-\left(\hat{\vec{p}}_{2}-\mathrm{e} \vec{A}\left(x_{2}\right)\right)^{2}\right]\left\langle\Psi_{\uparrow}^{+}\left(x_{1}\right) \Psi_{\uparrow}\left(x_{2}\right)\right\rangle \\
& =-g\left\langle\Psi_{\uparrow}^{+}\left(x_{1}\right) \Psi_{\downarrow}^{+}\left(x_{1}\right) \Psi_{\downarrow}\left(x_{1}\right) \Psi_{\uparrow}\left(x_{2}\right)\right\rangle+g\left\langle\Psi_{\uparrow}^{+}\left(x_{1}\right) \Psi_{\downarrow}^{+}\left(x_{2}\right) \Psi_{\downarrow}\left(x_{2}\right) \Psi_{\uparrow}\left(x_{2}\right)\right\rangle, \\
& \left\{\mathrm{i} \frac{\partial}{\partial t}-\mathrm{e} A_{0}\left(x_{1}\right)-\mathrm{e} A_{0}\left(x_{2}\right)\right\}\left\langle\Psi_{\downarrow}\left(x_{1}\right) \Psi_{\uparrow}\left(x_{2}\right)\right\rangle \\
& \quad-\frac{1}{2 m}\left[\left(\hat{\vec{p}}_{1}-\mathrm{e} \vec{A}\left(x_{1}\right)\right)^{2}+\left(\hat{\vec{p}}_{2}-\mathrm{e} \vec{A}\left(x_{2}\right)\right)^{2}\right]\left\langle\Psi_{\downarrow}\left(x_{1}\right) \Psi_{\uparrow}\left(x_{2}\right)\right\rangle \\
& =-g\left\langle\Psi_{\uparrow}^{+}\left(x_{1}\right) \Psi_{\downarrow}\left(x_{1}\right) \Psi_{\uparrow}\left(x_{1}\right) \Psi_{\uparrow}\left(x_{2}\right)\right\rangle+g\left\langle\Psi_{\downarrow}\left(x_{1}\right) \Psi_{\downarrow}^{+}\left(x_{2}\right) \Psi_{\downarrow}\left(x_{2}\right) \Psi_{\uparrow}\left(x_{2}\right)\right\rangle .
\end{aligned}
$$

The obtained system of equations of motion is not a closed one due to the correlation functions of higher order in these equations. In order to make the closed system of equations we use a disclosure of Hartree-Fock-Bogoliubov type (the meanfield approximation). For instance

$$
\begin{aligned}
\left\langle\Psi_{\uparrow}^{+}\left(x_{1}\right) \Psi_{\downarrow}^{+}\left(x_{1}\right) \Psi_{\downarrow}\left(x_{1}\right) \Psi_{\uparrow}\left(x_{2}\right)\right\rangle=\left\langle\Psi_{\uparrow}^{+}\left(x_{1}\right) \Psi_{\downarrow}^{+}\left(x_{1}\right)\right\rangle\left\langle\Psi_{\downarrow}\left(x_{1}\right) \Psi_{\uparrow}\left(x_{2}\right)\right\rangle \\
\quad+\left\langle\Psi_{\uparrow}^{+}\left(x_{1}\right) \Psi_{\uparrow}\left(x_{2}\right)\right\rangle\left\langle\Psi_{\downarrow}^{+}\left(x_{1}\right) \Psi_{\downarrow}\left(x_{1}\right)\right\rangle-\left\langle\Psi_{\uparrow}^{+}\left(x_{1}\right) \Psi_{\downarrow}\left(x_{1}\right)\right\rangle\left\langle\Psi_{\downarrow}^{+}\left(x_{1}\right) \Psi_{\uparrow}\left(x_{2}\right)\right\rangle,
\end{aligned}
$$

and similarly the other averages. After using the disclosure of type (6) the equation of motion (4), (5) will be as follows

$$
\begin{aligned}
& \left\{\mathrm{i} \frac{\partial}{\partial t}+\mathrm{e} A_{0}\left(x_{1}\right)-\mathrm{e} A_{0}\left(x_{2}\right)\right\}\left\langle\Psi_{\uparrow}^{+}\left(x_{1}\right) \Psi_{\uparrow}\left(x_{2}\right)\right\rangle \\
& \quad+\frac{1}{2 m}\left[\left(\hat{\vec{p}}_{1}+\mathrm{e} \vec{A}\left(x_{1}\right)\right)^{2}-\left(\hat{\vec{p}}_{2}-\mathrm{e} \vec{A}\left(x_{2}\right)\right)^{2}\right]\left\langle\Psi_{\uparrow}^{+}\left(x_{1}\right) \Psi_{\uparrow}\left(x_{2}\right)\right\rangle \\
& \quad=\Delta\left(x_{2}\right)\left\langle\Psi_{\uparrow}^{+}\left(x_{1}\right) \Psi_{\downarrow}^{+}\left(x_{2}\right)\right\rangle-\Delta^{*}\left(x_{1}\right)\left\langle\Psi_{\downarrow}\left(x_{1}\right) \Psi_{\uparrow}\left(x_{2}\right)\right\rangle, \\
& \left\{\mathrm{i} \frac{\partial}{\partial t}-\mathrm{e} A_{0}\left(x_{1}\right)-\mathrm{e} A_{0}\left(x_{2}\right)\right\}\left\langle\Psi_{\downarrow}\left(x_{1}\right) \Psi_{\uparrow}\left(x_{2}\right)\right\rangle \\
& -\frac{1}{2 m}\left[\left(\hat{\vec{p}}_{1}-\mathrm{e} \vec{A}\left(x_{1}\right)\right)^{2}+\left(\hat{\vec{p}}_{2}-\mathrm{e} \vec{A}\left(x_{2}\right)\right)^{2}\right]\left\langle\Psi_{\downarrow}\left(x_{1}\right) \Psi_{\uparrow}\left(x_{2}\right)\right\rangle \\
& =\Delta\left(x_{1}\right) \delta\left(\vec{r}_{1}-\vec{r}_{2}\right)-\Delta\left(x_{1}\right)\left\langle\Psi_{\uparrow}^{+}\left(x_{1}\right) \Psi_{\uparrow}\left(x_{2}\right)\right\rangle-\Delta\left(x_{2}\right)\left\langle\Psi_{\downarrow}^{+}\left(x_{2}\right) \Psi_{\downarrow}\left(x_{1}\right)\right\rangle .
\end{aligned}
$$

Here

$$
\Delta(x) \equiv g\left\langle\Psi_{\downarrow}(x) \Psi_{\uparrow}(x)\right\rangle
$$

is the order parameter. We note that in the obtained equations $(7),(8)$ the terms not relevant to the effect of superconductivity (Hartree and Fock terms) have been dropped. However, taking into account that the terms of Hartree type are trivial, one may include them into potential $A_{0}(x)$. 
The next step will be a separation of gauge-noninvariant multipliers (in fact we will use a reference system in which the condensate is motionless). Such a separation of phase has the following form [3]

$$
\begin{aligned}
\left\langle\Psi_{\sigma}^{+}\left(x_{1}\right) \Psi_{\sigma}\left(x_{2}\right)\right\rangle & =\exp \left\{\operatorname{im}\left(\chi\left(x_{2}\right)-\chi\left(x_{1}\right)\right)\right\} G_{\sigma}\left(x_{1}, x_{2}\right), \\
\left\langle\Psi_{\downarrow}(x) \Psi_{\uparrow}(x)\right\rangle & =\exp \left\{\operatorname{im}\left(\chi\left(x_{2}\right)+\chi\left(x_{1}\right)\right)\right\} F\left(x_{1}, x_{2}\right), \\
\Delta(x) & =\exp \{2 \operatorname{im} \chi(x)\}|\Delta(x)| .
\end{aligned}
$$

Then the equations of motion for $G$ and $F$ are as follows:

$$
\begin{aligned}
& \left\{\mathrm{i} \frac{\partial}{\partial t}+\frac{1}{2 m}\left[\left(\hat{\vec{p}}_{1}-m \vec{v}_{s}\left(x_{1}\right)\right)^{2}-\left(\hat{\vec{p}}_{2}+m \vec{v}_{s}\left(x_{2}\right)\right)^{2}\right]\right\} G_{\uparrow}\left(x_{1}, x_{2}\right) \\
& +\left\{m \dot{\chi}\left(x_{1}\right)-m \dot{\chi}\left(x_{2}\right)+\mathrm{e} A_{0}\left(x_{1}\right)-\mathrm{e} A_{0}\left(x_{2}\right)\right\} G_{\uparrow}\left(x_{1}, x_{2}\right) \\
& =\left|\Delta\left(x_{2}\right)\right| F^{*}\left(x_{2}, x_{1}\right)-\left|\Delta\left(x_{1}\right)\right| F\left(x_{1}, x_{2}\right), \\
& \left\{\mathrm{i} \frac{\partial}{\partial t}-\frac{1}{2 m}\left[\left(\hat{\vec{p}}_{1}+m \vec{v}_{s}\left(x_{1}\right)\right)^{2}+\left(\hat{\vec{p}}_{2}+m \vec{v}_{s}\left(x_{2}\right)\right)^{2}\right]\right\} F\left(x_{1}, x_{2}\right) \\
& -\left\{m \dot{\chi}\left(x_{1}\right)+m \dot{\chi}\left(x_{2}\right)+\mathrm{e} A_{0}\left(x_{2}\right)+\mathrm{e} A_{0}\left(x_{1}\right)\right\} F\left(x_{1}, x_{2}\right) \\
& =\left|\Delta\left(x_{1}\right)\right| \delta\left(\vec{r}_{1}-\vec{r}_{2}\right)-\left|\Delta\left(x_{1}\right)\right| G_{\uparrow}\left(x_{1}, x_{2}\right)-\left|\Delta\left(x_{2}\right)\right| G_{\downarrow}\left(x_{2}, x_{1}\right) \text {, }
\end{aligned}
$$

where

$$
\vec{v}_{s}=\nabla \chi-\frac{e}{m} \vec{A}
$$

is superfluid velocity (velocity of the condensate). It is a gauge-invariant combination, because a change of calibration may be compensated by a gauge transformation of the vector potential.

The transition to equations of hydrodynamics is performed using an expansion of equations (11) and (12) in terms of space gradients. This expansion can be simply performed by using the so-called mixed Wigner representation [3]. To this end we introduce new variables

$$
\vec{R}=\frac{1}{2}\left(\vec{r}_{1}+\vec{r}_{2}\right), \quad \vec{r}=\vec{r}_{2}-\vec{r}_{1} .
$$

After the Fourier transformation with respect to relative coordinate $\vec{r}$ we obtain

$$
f\left(x_{1}, x_{2}\right) \rightarrow f(\vec{R}, \vec{r}, t)=\int \frac{\mathrm{d} \vec{p}}{(2 \pi)^{3}} f(\vec{R}, \vec{p}, t) \mathrm{e}^{\mathrm{i} p \vec{p}},
$$

and

$$
\begin{array}{ll}
\vec{r}_{1} \rightarrow \vec{R}-\frac{\mathrm{i}}{2} \nabla_{\vec{p}}, & \vec{r}_{2} \rightarrow \vec{R}+\frac{\mathrm{i}}{2} \nabla_{\vec{p}}, \\
\hat{\vec{p}}_{1} \rightarrow \vec{p}-\frac{\mathrm{i}}{2} \nabla_{\vec{R}}, & \hat{\vec{p}}_{2} \rightarrow-\vec{p}-\frac{\mathrm{i}}{2} \nabla_{\vec{R}} .
\end{array}
$$

Any function of $\vec{R}+\mathrm{i} / 2 \cdot \nabla_{\vec{p}}$ can be understood in terms of its power-series expansion

$$
f\left(\vec{R}+\frac{\mathrm{i}}{2} \nabla_{\vec{p}}\right)=f(\vec{R})+\frac{\mathrm{i}}{2} \frac{\partial f(\vec{R})}{\partial \vec{R}} \frac{\partial}{\partial \vec{p}}-\cdots
$$


Using procedures (13) and (14) the equations for correlation functions can be written as follows

$$
\begin{gathered}
\mathrm{i}\left\{\frac{\partial}{\partial t}+\mathrm{e} E_{i} \frac{\partial}{\partial p_{i}}-m \dot{v}_{s i} \frac{\partial}{\partial p_{i}}-\frac{\partial}{\partial R_{j}}\left(\frac{\left(p_{i}+m v_{s i}\right)^{2}}{2 m}\right) \frac{\partial}{\partial p_{j}}\right. \\
\left.\quad+\left(\frac{p_{i}}{m}+v_{s i}\right) \frac{\partial}{\partial R_{i}}\right\} G_{\uparrow}(\vec{R}, \vec{p}, t)=|\Delta(\vec{R}, t)|\left(F^{*}(\vec{R}, \vec{p}, t)-F(\vec{R}, \vec{p}, t)\right) \\
\quad-\frac{\mathrm{i}}{2} \frac{\partial|\Delta(\vec{R}, t)|}{\partial R_{i}} \frac{\partial}{\partial p_{i}}\left(F^{*}(\vec{R}, \vec{p}, t)+F(\vec{R}, \vec{p}, t)\right), \\
\left\{\mathrm{i} \frac{\partial}{\partial t}-2 \mathrm{e} A_{0}(\vec{R}, t)-2 m \dot{\chi}(\vec{R}, t)-\mathrm{i}\left(p_{i} \frac{\partial v_{s i}}{\partial R_{j}} \frac{\partial}{\partial p_{j}}-v_{s i} \frac{\partial}{\partial R_{i}}\right)\right. \\
\left.-\left(\frac{p^{2}}{m}+v_{s}^{2}\right)\right\} F(\vec{R}, \vec{p}, t)=|\Delta(\vec{R}, t)|\left(1-G_{\uparrow}(\vec{R}, \vec{p}, t)-G_{\downarrow}(\vec{R},-\vec{p}, t)\right) \\
\quad-\frac{\mathrm{i}}{2} \frac{\partial|\Delta(\vec{R}, t)|}{\partial R_{i}} \frac{\partial}{\partial p_{i}}\left(G_{\uparrow}(\vec{R}, \vec{p}, t)-G_{\downarrow}(\vec{R},-\vec{p}, t)\right) .
\end{gathered}
$$

In the obtained equations the second order terms with respect to space gradient (the terms proportional to $\nabla_{\vec{R}}^{2}$ ) were neglected.

\section{Two-fluid hydrodynamics}

By solving equations (15), (16) we use the perturbation theory. Therefore, we have to determine the order of other terms in these equations.

Let $L$ be the length, on which the macroscopic quantities can change (it scales with the size of the system). Then

$$
\left|\nabla_{\vec{R}}\right| \sim L^{-1}, \quad \frac{\partial}{\partial t} \sim \bar{u} L^{-1} \sim v_{\mathrm{F}} \frac{T_{\mathrm{c}}}{E_{\mathrm{F}}} L^{-1}
$$

where $T_{\mathrm{c}}$ is critical temperature, $E_{\mathrm{F}}=\left(m v_{\mathrm{F}}^{2}\right) / 2=\left(p_{\mathrm{F}}^{2}\right) /(2 m)$ is Fermi energy. The characteristic momenta are of the order of Fermi momentum, therefore we can put

$$
p=p_{\mathrm{F}}+\frac{\xi}{v_{\mathrm{F}}}, \quad \xi \sim T_{\mathrm{c}} .
$$

Then

$$
\left|\nabla_{\vec{p}}\right| \sim \frac{v_{\mathrm{F}}}{T_{\mathrm{c}}} \sim \xi_{0} \sim 10^{-4} \mathrm{~cm}
$$

where $\xi_{0}$ is the coherence length. The gap $\Delta$ is of the order $T_{\mathrm{c}}$.

This theory has two small parameters. It is $\xi_{0} / L$ and $a / \xi_{0}=T_{\mathrm{c}} / E_{\mathrm{F}}$, where $a$ is the interatomic distance. The first parameter is associated with the hydrodynamic approach, while the second is semi-classical. Hereinafter the semi-classical motion of electrons will be neglected. Therefore, we may put $T_{\mathrm{c}} \sim E_{\mathrm{F}}$.

We denote

$$
\alpha=\frac{\xi_{0}}{L} \ll 1
$$


Dividing equations (15) and (16) by $T_{\mathrm{c}}$ and using (18) we get

$$
\begin{aligned}
\mathrm{i} \alpha\left\{\frac{\partial}{\partial t}+\right. & \mathrm{e} E_{i} \frac{\partial}{\partial p_{i}}-m \dot{v}_{s i} \frac{\partial}{\partial p_{i}}-\frac{\partial}{\partial R_{j}}\left(\frac{\left(p_{i}+m v_{s i}\right)^{2}}{2 m}\right) \frac{\partial}{\partial p_{j}} \\
& \left.+\left(\frac{p_{i}}{m}+v_{s i}\right) \frac{\partial}{\partial R_{i}}\right\} G_{\uparrow}(\vec{R}, \vec{p}, t)=|\Delta(\vec{R}, t)|\left(F^{*}(\vec{R}, \vec{p}, t)-F(\vec{R}, \vec{p}, t)\right) \\
& -\alpha \frac{\mathrm{i}}{2} \frac{\partial|\Delta(\vec{R}, t)|}{\partial R_{i}} \frac{\partial}{\partial p_{i}}\left(F^{*}(\vec{R}, \vec{p}, t)+F(\vec{R}, \vec{p}, t)\right), \\
\left\{\mathrm{i} \alpha \frac{\partial}{\partial t}-\right. & 2 e A_{0}(\vec{R}, t)-2 m \dot{\chi}(\vec{R}, t)-\mathrm{i} \alpha\left(p_{i} \frac{\partial v_{s i}}{\partial R_{j}} \frac{\partial}{\partial p_{j}}-v_{s i} \frac{\partial}{\partial R_{i}}\right) \\
& \left.-\left(\frac{p^{2}}{m}+v_{s}^{2}\right)\right\} F(\vec{R}, \vec{p}, t)=|\Delta(\vec{R}, t)|\left(1-G_{\uparrow}(\vec{R}, \vec{p}, t)-G_{\downarrow}(\vec{R},-\vec{p}, t)\right) \\
& -\alpha \frac{\mathrm{i}}{2} \frac{\partial|\Delta(\vec{R}, t)|}{\partial R_{i}} \frac{\partial}{\partial p_{i}}\left(G_{\uparrow}(\vec{R}, \vec{p}, t)-G_{\downarrow}(\vec{R},-\vec{p}, t)\right) .
\end{aligned}
$$

In order to solve the equations (19) and (20) we formally expand the functions $G, F$ and $\Delta$ in powers of $\alpha$

$$
f=f^{(0)}+\alpha f^{(1)}
$$

In the lowest order equation (19) gives

$$
F^{(0)}{ }^{*}(\vec{R}, \vec{p}, t)=F^{(0)}(\vec{R}, \vec{p}, t),
$$

that means a real function $F^{(0)}$. The equation (20) by using (22) in the lowest order gives

$$
\begin{aligned}
\left(\mathrm{e} A_{0}(\vec{R}, t)\right. & \left.+m \dot{\chi}(\vec{R}, t)+\frac{1}{2} m v_{s}^{2}(\vec{R}, t)\right) F^{(0)}(\vec{R}, \vec{p}, t) \\
= & -\frac{p^{2}}{2 m} F^{(0)}(\vec{R}, \vec{p}, t)-\frac{1}{2}\left|\Delta^{(0)}(\vec{R}, t)\right|\left(1-G_{\uparrow}^{(0)}(\vec{R}, \vec{p}, t)-G_{\downarrow}^{(0)}(\vec{R},-\vec{p}, t)\right) .
\end{aligned}
$$

The separation of variables gives

$$
\mathrm{e} A_{0}(\vec{R}, t)+m \dot{\chi}(\vec{R}, t)+\frac{1}{2} m v_{s}^{2}(\vec{R}, t)+\mu(\vec{R}, t)=0
$$

and

$$
2 \xi_{p} F^{(0)}(\vec{R}, \vec{p}, t)+\left|\Delta^{(0)}(\vec{R}, t)\right|\left(1-G_{\uparrow}^{(0)}(\vec{R}, \vec{p}, t)-G_{\downarrow}^{(0)}(\vec{R},-\vec{p}, t)\right),
$$

where $\xi_{p}=p^{2} /(2 m)-\mu$ and $\mu$ - is the order of separation of variables. In the local equilibrium state $\mu$ - is chemical potential and equation (25) is reduced to the equation for order parameter.

Applying the operation $\nabla_{\vec{R}}$ to equation (24) we obtain the equation of motion for superfluid velocity

$$
m \frac{\partial \vec{v}_{s}}{\partial t}+\nabla_{\vec{R}}\left(\frac{m \vec{v}_{s}^{2}}{2}+\mu\right)=\mathrm{e} \vec{E}
$$


By using the vector identity

$$
\frac{1}{2} \nabla \vec{v}_{s}^{2}=\vec{v}_{s} \times\left(\nabla \times \vec{v}_{s}\right)+\vec{v}_{s} \cdot \nabla \vec{v}_{s}=-\frac{\mathrm{e}}{m} \vec{v}_{s} \times \overrightarrow{\mathcal{H}}+\vec{v}_{s} \cdot \nabla \vec{v}_{s}
$$

Equation (15) can be written as follows

$$
m \frac{\mathrm{d} \vec{v}_{s}}{\mathrm{~d} t}=\mathrm{e}\left[\vec{E}+\left(\vec{v}_{s} \times \overrightarrow{\mathcal{H}}\right)\right]-\nabla \mu
$$

This is the first hydrodynamic equation and shows that the superfluid accelerates freely under the applied fields. The remaining hydrodynamic equations are provided by the conservation relations for the particle density $\rho(\vec{R}, t)$, momentum density $\vec{j}(\vec{R}, t)$ and energy density $\mathcal{E}(\vec{R}, t)$.

Let us consider the first order of equations (19) and (20). These equations are as follows:

$$
\begin{aligned}
& \frac{\partial G_{\uparrow}^{(0)}(\vec{R}, \vec{p}, t)}{\partial t}+\left(\frac{p_{i}}{m}+v_{s i}\right) \frac{\partial G_{\uparrow}^{(0)}(\vec{R}, \vec{p}, t)}{\partial R_{i}}+\frac{\partial G_{\uparrow}^{(0)}(\vec{R}, \vec{p}, t)}{\partial p_{i}} \\
& \times\left(e E_{i}-m \dot{v}_{s i}-\frac{\partial}{\partial R_{i}}\left(\frac{\left(\vec{p}+m \vec{v}_{s}\right)^{2}}{2 m}\right)\right)-\frac{\partial \Delta^{(0)}(\vec{R}, t)}{\partial R_{i}} \frac{\partial F^{(0)}(\vec{R}, \vec{p}, t)}{\partial p_{i}} \\
&=2\left|\Delta^{(0)}(\vec{R}, t)\right| \operatorname{Im} F^{(1)}(\vec{R}, \vec{p}, t), \\
& \frac{\partial F^{(0)}(\vec{R}, \vec{p}, t)}{\partial t}+2 \mathrm{i}\left(\mathrm{e} A_{0}(\vec{R}, t)+m \dot{\chi}(\vec{R}, t)+\frac{1}{2} m v_{s}^{2}(\vec{R}, t)+\frac{p^{2}}{2 m}\right) F^{(1)}(\vec{R}, \vec{p}, t) \\
&=\frac{\partial \Delta^{(0)}(\vec{R}, t)}{\partial R_{j}} \frac{\partial}{\partial p_{j}}\left(G_{\uparrow}^{(0)}(\vec{R}, \vec{p}, t)-G_{\downarrow}^{(0)}(\vec{R},-\vec{p}, t)\right)+\frac{\partial}{\partial p_{j}}\left(p_{i} \frac{\partial v_{s i}}{\partial R_{j}} F^{(0)}(\vec{R}, \vec{p}, t)\right) \\
&-\frac{\partial}{\partial R_{i}}\left(v_{s i} F^{(0)}(\vec{R}, \vec{p}, t)\right)-\mathrm{i}\left|\Delta^{(1)}(\vec{R}, t)\right|\left(1-G_{\uparrow}^{(0)}(\vec{R}, \vec{p}, t)-G_{\downarrow}^{(0)}(\vec{R},-\vec{p}, t)\right) \cdot(29)
\end{aligned}
$$

The relations for $\rho, \vec{j}$ and $\mathcal{E}$ follow simply from calculation of moments of (28) and (29)(see [6]). By definition

$$
\rho(\vec{R}, t)=2 m \int \frac{\mathrm{d} \vec{p}}{(2 \pi)^{3}} G_{\uparrow}^{(0)}(\vec{R}, \vec{p}, t)
$$

and after integrating (28) over $\vec{p}$ we find

$$
\frac{\partial \rho}{\partial t}+\operatorname{div} \vec{j}=0
$$

where

$$
\vec{j}(\vec{R}, t)=2 \int \frac{\mathrm{d} \vec{p}}{(2 \pi)^{3}} \vec{p} G_{\uparrow}^{(0)}(\vec{R}, \vec{p}, t)+\rho \vec{v}_{s} \equiv \vec{j}_{0}+\rho \vec{v}_{s}
$$


By analogy, a simple calculation gives

$$
\frac{\partial j_{k}}{\partial t}+\frac{\partial \Pi_{i k}}{\partial R_{i}}=\frac{\mathrm{e}}{m} \rho E_{k}+\frac{\mathrm{e}}{m c} H_{k i} j_{i}
$$

where $H_{k i}$ is magnetic field intensity tensor and stress tensor $\Pi_{i k}$ is given by

$$
\Pi_{i k}=\frac{2}{m} \int \frac{\mathrm{d} \vec{p}}{(2 \pi)^{3}}\left(p_{i}+m v_{s i}\right)\left(p_{k}+m v_{s k}\right) G_{\uparrow}^{(0)}(\vec{R}, \vec{p}, t)+\delta_{i k} \frac{\Delta^{(0)}(\vec{R}, t)}{g} .
$$

The energy density (without mean-field energy) is

$$
\mathcal{E}(\vec{R}, t)=\frac{1}{m} \int \frac{\mathrm{d} \vec{p}}{(2 \pi)^{3}}\left(\vec{p}+m \overrightarrow{v_{s}}\right)^{2} G_{\uparrow}^{(0)}(\vec{R}, \vec{p}, t)+\frac{\left|\Delta^{(0)}(\vec{R}, t)\right|^{2}}{g},
$$

or

$$
\mathcal{E}=\mathcal{E}_{0}+\vec{j}_{0} \vec{v}_{s}+\frac{1}{2} \rho v_{s}^{2}
$$

where

$$
\mathcal{E}_{0}=\frac{1}{m} \int \frac{\mathrm{d} \vec{p}}{(2 \pi)^{3}} p^{2} G_{\uparrow}^{(0)}(\vec{R}, \vec{p}, t)+\frac{\left|\Delta^{(0)}(\vec{R}, t)\right|^{2}}{g} .
$$

Using (28), (29) and (24) we obtain

$$
\frac{\partial \mathcal{E}}{\partial t}+\operatorname{div} \vec{Q}=\frac{\mathrm{e}}{m} \vec{E} \vec{j}
$$

the energy current is given by

$$
\vec{Q}=\int \frac{\mathrm{d} \vec{p}}{(2 \pi)^{3}}\left(\vec{p}+m \vec{v}_{s}\right)\left(\frac{\vec{p}}{m}+\vec{v}_{s}\right)^{2} G_{\uparrow}^{(0)}(\vec{R}, \vec{p}, t)+2 \frac{\Delta^{(0)}(\vec{R}, t)}{g} \vec{v}_{s} .
$$

The flows of hydrodynamic quantities may be calculated if we assume that in zeroth approximation the thermodynamic local equilibrium is provided by the gradients. In local equilibrium

$$
G_{\uparrow}^{(0)}=v_{\vec{p}}^{2}+u_{\vec{p}}^{2} f\left(\frac{\varepsilon_{\vec{p}}-\vec{u} \vec{p}}{T}\right)-v_{\vec{p}}^{2} f\left(\frac{\varepsilon_{\vec{p}}+\vec{u} \vec{p}}{T}\right),
$$

where

$f(x)=\left(\mathrm{e}^{x}+1\right)^{-1}, \quad u_{\vec{p}}^{2}=\frac{1}{2}\left(1+\frac{\xi_{\vec{p}}}{\varepsilon_{\vec{p}}}\right), \quad v_{\vec{p}}^{2}=\frac{1}{2}\left(1-\frac{\xi_{\vec{p}}}{\varepsilon_{\vec{p}}}\right), \quad \varepsilon_{\vec{p}}=\sqrt{\xi_{\vec{p}}^{2}+\Delta^{2}}$.

Using expression (37) we obtain

$$
\begin{aligned}
& \vec{j}=\rho_{s} \vec{v}_{s}+\rho_{n} \vec{v}_{n}, \quad \vec{v}_{n} \equiv \vec{u}+\vec{v}_{s}, \\
& \Pi_{i k}=\rho_{n} v_{n i} v_{n k}+\rho_{s} v_{s i} v_{s k}+\delta_{i k} P \\
& \vec{Q}=\left(\frac{v_{s}^{2}}{2}+\frac{\mu}{m}\right) \vec{j}+T S \vec{v}_{n}+\rho_{n} \vec{v}_{n}\left(\vec{v}_{n} \cdot\left(\vec{v}_{n}-\vec{v}_{s}\right)\right) .
\end{aligned}
$$


Here

$$
\rho_{n}=\frac{1}{u^{2}} \int \frac{\mathrm{d} \vec{p}}{(2 \pi)^{3}} \vec{p} \vec{u} f\left(\frac{\varepsilon_{\vec{p}}-\vec{u} \vec{p}}{T}\right)
$$

is normal density,

$$
\rho_{s}=\rho-\rho_{n}
$$

is superfluid density,

$$
P=T S-\mathcal{E}_{0}+u^{2} \rho_{n}+\rho \mu / m
$$

is pressure, and entropy is represented as follows:

$$
S=2 \int \frac{\mathrm{d} \vec{p}}{(2 \pi)^{3}} \ln \left(1+\exp \left(\frac{\varepsilon_{\vec{p}}-\vec{u} \vec{p}}{T}\right)\right)+\frac{2}{T} \int \frac{\mathrm{d} \vec{p}}{(2 \pi)^{3}} f\left(\frac{\varepsilon_{\vec{p}}-\vec{u} \vec{p}}{T}\right) .
$$

The equations (27), (31), (32), (35) and (38) form a complete system of magnetohydrodynamic equations for superconductor.

\section{References}

1. Nozières P., Pines D. The Theory of Quantum Liquids. Addison-Wesley, Redwood City, California, 1990.

2. Yakovlev D.G., Levenfish K.P., Shibanov Yu.A., Usp. Fiz. Nauk, 1999, 169, 825 (in Russian).

3. Svidzynsky A.V. Microscopic Theory of Superconductivity. Lutsk, Veza, 2001 (In Ukrainian).

4. Landau L.D., JETF, 1941, 11, 592 (in Russian).

5. Bogolubov N.N. To the question about hydrodynamics of superfluid liquid. Preprint UINR, Dubna, 1963 (in Russian).

6. Svidzynsky A.V. The Space Inhomogenous Problems Theory of Superconductivity. Moscow, Nauka, 1982 (in Russian).

7. Stephen M.J. Phys. Rev. A, 1965, 139, 197. 


\title{
Мікроскопічне виведення рівнянь гідродинаміки для надплинних фермі систем
}

\author{
А.Свідзинський, П.Шигорін \\ Волинський державний університет ім. Лесі Українки
}

Отримано 3 серпня 2005 р.

Виходячи з перших принципів статистичної механіки, побудовано дворідинну гідродинаміку надпровідника в ідеальному наближенні. Для побудови гідродинаміки використано систему рівнянь руху для нормальної та аномальної кореляційних функцій. Перехід до рівнянь гідродинаміки здійснюється через розклад рівнянь руху для кореляційних функцій за малим параметром.

Ключові слова: дворідинна гідродинаміка, кореляційна функція, надплинні Фермі-системи

PACS: 05.30.Fk, 47.37.+q 